\title{
Analysis of Dynamic Cross Response between Spindles in a Dual Spindle Type Multi-Functional Turning Machine
}

\author{
Y. H. Choi ${ }^{1}$, S. T. Kim ${ }^{1}$, T. Y. Seo ${ }^{2}$, K. H. Kim ${ }^{3}$ \\ ${ }^{1}$ Department of Mechanical Engineering, Changwon National University, Changwon, Korea; ${ }^{2}$ Department of Mechanical Design Engi- \\ neering, Graduate School of Changwon National University, Changwon, Korea; ${ }^{3}$ Technology Research Institute, AMECO Co., Ltd., \\ Korea. \\ Email: yhchoi@changwon.ac.kr
}

Received October 2013

\begin{abstract}
In order to meet increasing demand for higher productivity and flexibility, recently many kinds of multi-functional machine tools, which are capable of multiple machining functions or different kinds of machining processes on one machine, have been developed and widely used in manufacturing industries. In this study, a multi-functional turning lathe, which has two spindles and two turrets so that multiple turning operations and various machining processes could be performed simultaneously, has been developed. Furthermore, the equations of correlation between whole responses and cross responses of the two spindles have been derived to examine to what extent the two spindles affect each other's vibrations.
\end{abstract}

Keywords: Multi-Functional Lathe; Multi-Spindles; Frequency Response; Cross Response; FEM Structural Analysis

\section{Introduction}

In the last couple of decades, as the demands are increasing to produce machine parts with higher productivity and accuracy at reduced cost, many researches and developments on multi-functional machine tools have been performed [1-5,10,11]. Nowadays multi-functional machine tools are widely used to machining various mechanical components in the aerospace, automobile, power plant industries and so on. Figure 1 shows a brief history of the advancement in the configuration of turning center (TC) [1]. And Figure 2 shows typical examples of machined parts as the functionality of TC was increased [3].

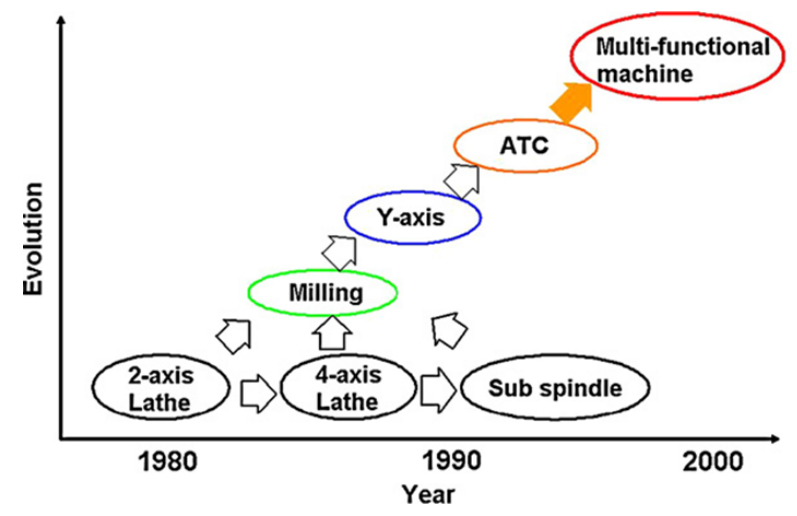

Figure 1. Evolution of turning machines (after T. Moriwaki [1]).
The machine tool shown in Figure $\mathbf{3}$ is a multi-functional turning lathe under developing for machining complex automotive parts. It consists of 2 spindles and 2 turrets so that various machining process could be performed simultaneously. Regarding a multi-functional turning lathe that consists of two spindles, the two spindles operating simultaneously may affect each other because of interactions between their cutting forces. Thus resulting machining accuracy may be worse than that of a single spindle only machining.

Vibration is one of the dominant causes that most badly affect the machining accuracy of machine tools [6, 7]. In order to examine how the two spindles affect each other's vibrations when they are operating simultaneous-

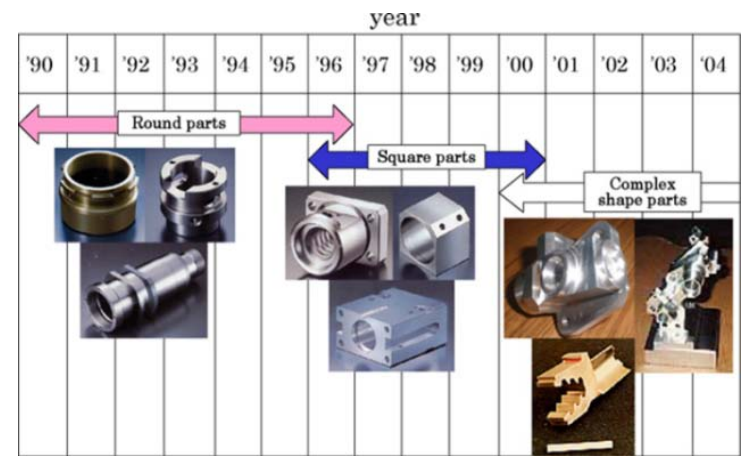

Figure 2. Evolution of parts machined by turning machines (after T. Moriwaki [1]). 


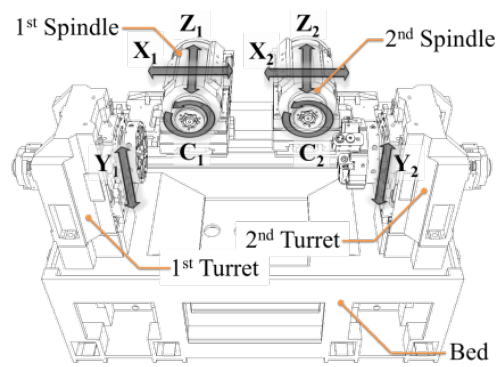

Figure 3. A multi-functional turning lathe for machining automobile parts.

ly, in this study, the equations of correlation between whole responses and cross responses of the two spindles have been derived and FEM harmonic response analysis has been carried out of the multi-functional turning lathe. A generalized machine structure model has been introduced for deriving the correlation equations.

\section{Cross Response Analysis}

\subsection{A Generalized Machine Model Analysis}

In order to derive the correlation equation between responses of at arbitrary two different nodes on a machine structure acted on by excitation forces, in this study, a generalized machine structure model is introduced as shown in Figure 4. For the generalized machine model of multi-degree-of-freedom (DOF) shown in Figure 4, the displacement response, $\{\mathbf{D}\}$ due to the applied force vector, $\{\mathbf{F}\}$ can be determined from following transfer function relationship.

$$
\{\mathbf{D}\}=[\mathbf{H}(i \omega)]\{\mathbf{F}\}
$$

Where $\{\mathbf{D}\}=\left(\mathbf{D}_{1}, \mathbf{D}_{2}\right)^{T}=\left(X_{1}, Y_{1}, Z_{1}, X_{2}, Y_{2}, Z_{2}\right)^{T}$ is a displacement vector condensed on nodes 1 and 2 , $\{\mathbf{F}\}=\left(\mathbf{F}_{1}, \mathbf{F}_{2}\right)^{T}=\left(F_{1 X}, F_{1 Y}, F_{1 Z}, F_{2 X}, F_{2 Y}, F_{2 Z}\right)^{T}$ is a applied force vector, $[\mathbf{H}(i \omega)]=\left[-\omega^{2}[\mathbf{M}]+i \omega[\mathbf{C}]+[\mathbf{K}]\right]^{-1}$ is a transfer function, $[\mathbf{M}],[\mathbf{C}],[\mathbf{K}]$ are mass, stiffness, and equivalent viscous damping coefficient matrices, respectively. And the symbol $\omega$ denotes angular frequency. Equation (1) can be denoted as Equation (2) by identifying nodal degree of freedom.

$$
\left\{\begin{array}{l}
\mathbf{D}_{1} \\
\mathbf{D}_{2}
\end{array}\right\}=\left[\begin{array}{ll}
\mathbf{H}_{11} & \mathbf{H}_{12} \\
\mathbf{H}_{21} & \mathbf{H}_{22}
\end{array}\right]\left\{\begin{array}{l}
\mathbf{F}_{1} \\
\mathbf{F}_{2}
\end{array}\right\}
$$

Where, sub-matrices $\mathbf{H}_{i j}$ are defined as followings.

$$
\begin{aligned}
\mathbf{H}_{11} & =\left[\begin{array}{lll}
h_{11} & h_{12} & h_{13} \\
h_{21} & h_{22} & h_{23} \\
h_{31} & h_{32} & h_{33}
\end{array}\right] \\
\mathbf{H}_{12} & =\left[\begin{array}{lll}
h_{14} & h_{15} & h_{16} \\
h_{24} & h_{25} & h_{26} \\
h_{34} & h_{35} & h_{36}
\end{array}\right]
\end{aligned}
$$

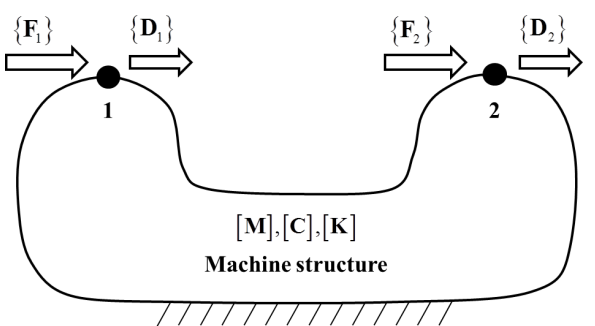

Figure 4. A generalized multi-DOF machine structure model acted on by dynamic forces at two different nodes.

$$
\begin{array}{r}
\mathbf{H}_{21}=\left[\begin{array}{lll}
h_{41} & h_{42} & h_{43} \\
h_{51} & h_{52} & h_{53} \\
h_{61} & h_{62} & h_{63}
\end{array}\right] \\
\mathbf{H}_{22}=\left[\begin{array}{lll}
h_{44} & h_{45} & h_{46} \\
h_{54} & h_{55} & h_{56} \\
h_{64} & h_{65} & h_{66}
\end{array}\right]
\end{array}
$$

In Equations (3-a)-(3-d), if the sub-matrices $\mathbf{H}_{i j}$ were assumed as diagonal matrices, which seems to be rational assumption in most mechanical structures of isotropic materials, the whole frequency responses at nodes 1 and 2 can be obtained, respectively as follows.

$$
\begin{gathered}
X_{1}=h_{11} F_{1 X}+h_{14} F_{2 X}=X_{11}+X_{12} \\
Y_{1}=h_{22} F_{1 Y}+h_{25} F_{2 Y}=Y_{11}+Y_{12} \\
Z_{1}=h_{33} F_{1 Z}+h_{36} F_{2 Z}=Z_{11}+Z_{12} \\
X_{2}=h_{44} F_{2 X}+h_{41} F_{1 X}=X_{22}+X_{21} \\
Y_{2}=h_{55} F_{2 Y}+h_{52} F_{1 Y}=Y_{22}+Y_{21} \\
Z_{2}=h_{66} F_{2 Z}+h_{63} F_{1 Z}=Z_{22}+Z_{21}
\end{gathered}
$$

Where, $X_{11}, Y_{11}, Z_{11}$ and $X_{22}, Y_{22}, Z_{22}$ are auto-responses that are $X$-, $Y$-, $Z$-responses at nodes 1 and 2, respectively due to the forces applied to the same node. $X_{12}, Y_{12}, Z_{12}$ and $X_{21}, Y_{21}, Z_{21}$ are cross responses that are the displacements brought about at one node due to the associated forces acting on the other node.

Based upon the correlation Equations (4-a)-(4-f), the static cross response to total response ratios (SCR) in the $X$-, $Y$-, Z-directions are defined as follows, respectively.

$$
\begin{gathered}
S_{C C R_{X i j}}=\left(\frac{X_{i j}}{X_{i}}\right)_{\omega=0} \quad i=1,2 \text { and } j=2,1 \\
S C R_{Y i j}=\left(\frac{Y_{i j}}{Y_{i}}\right)_{\omega=0} \quad i=1,2 \text { and } j=2,1 \\
S C R_{Z i j}=\left(\frac{Z_{i j}}{Z_{i}}\right)_{\omega=0} \quad i=1,2 \text { and } j=2,1
\end{gathered}
$$

Similarly the dynamic cross response to total response ratios (DCR) in the $X-, Y-, Z$-directions are defined as 
follows, respectively.

$$
\begin{aligned}
D C R_{X i j}=\left(\frac{X_{i j}}{X_{i}}\right)_{\max } \quad i=1,2 \text { and } j=2,1 \\
D C R_{Y i j}=\left(\frac{Y_{i j}}{Y_{i}}\right)_{\max } \quad i=1,2 \text { and } j=2,1 \\
D C R_{Z i j}=\left(\frac{Z_{i j}}{Z_{i}}\right)_{\max } \quad i=1,2 \text { and } j=2,1
\end{aligned}
$$

Where subscript "max" means "the peak resonance frequency where the maximum harmonic response occurs". The percent SCR and the percent DCR can be obtained from SCR and DCR multiplied by 100, respectively.

\subsection{Cross Response Analysis between Spindles of a Multi-Functional Turning Machine}

In order to obtain SCRs, DCRs, and percent SCRs, percent DCRs at the $1^{\text {st }}$ and $2^{\text {nd }}$ spindles of the multi-functional lathe, FEM harmonic response analysis has been carried out. Finite element model is presented in Figure 5 and modeling data are listed in the Table 1. In case of the FEM model shown in Figure 5 of the multi-functional turning lathe, node numbers $i, j$ correspond to the spindle numbers. Prior to this FEM analysis, the multifunctional turning lathe shown in Figure 5 had been optimized for lightweight and high rigidity [8,9,11].

As the result of FEM harmonic response analysis, harmonic frequency responses of the multi-functional turning lathe are obtained; total responses $\left(X_{i}, Y_{i}, Z_{i}\right)$, au- to-responses $\left(X_{i i}, Y_{i i}, Z_{i i}\right)$, and cross-responses $\left(X_{i j}, Y_{i j}\right.$, $Z_{i j}$ ) at the $i$-th spindle. The responses computed at each spindle are illustrated in Figures 6 and 7. As seen from Figures 6 and 7, it is apparent that almost all of both percent SCRs and percent DCRs are less than 5\% except the $1^{\text {st }}$ spindle's percent DCR, which is above $10 \%$. As stated above, static response means the response at the frequency $\omega=0$ and dynamic response (peak response) designate the maximum response among resonant peak responses.

Applied the Equations (5) and (6) with the FEM harmonic analysis results, both the percent SCRs and the percent DCRs at the $1^{\text {st }}$ spindle have been determined and listed in Table 2.

Similarly, the percent SCRs and the percent DCRs at the $2^{\text {nd }}$ spindle also have been obtained and summarized in Table 3.

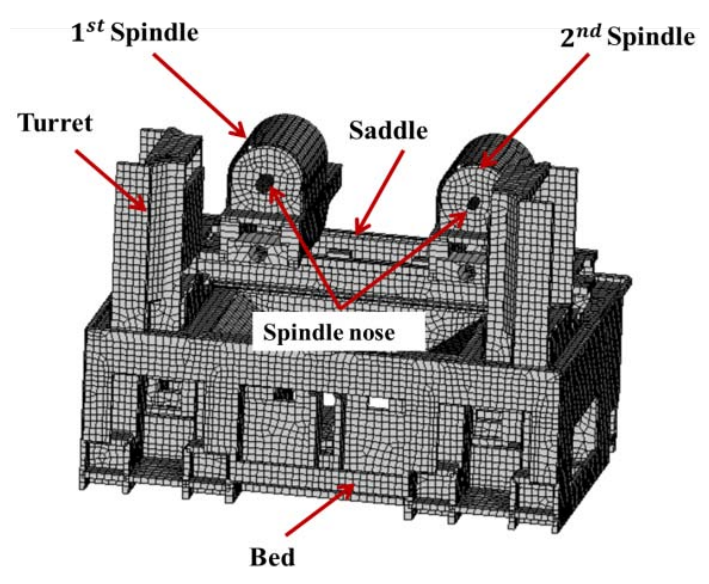

Figure 5. FEM model of the multi-functional lathe.

Table 1. Modeling data for FEM structural analysis.

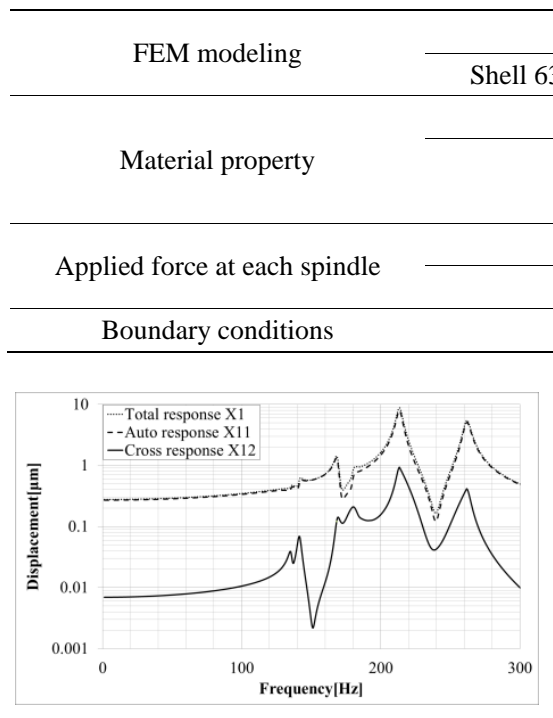

(a) In the $X$-direction

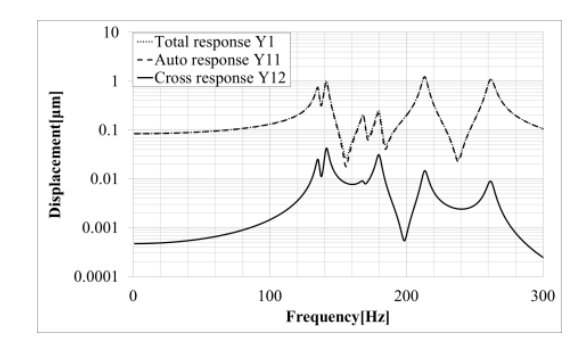

(b) In the $Y$-direction

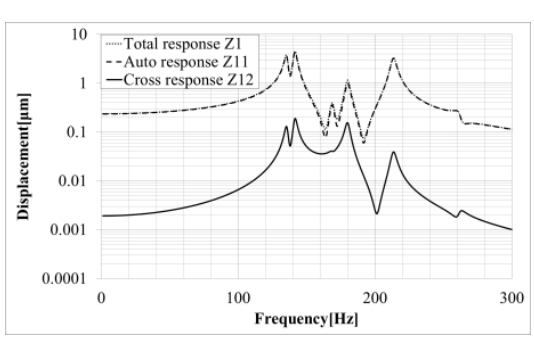

(c) In the Z-direction

Figure 6. Computed harmonic responses, auto- and cross-responses at the $1^{\text {st }}$ spindle. 


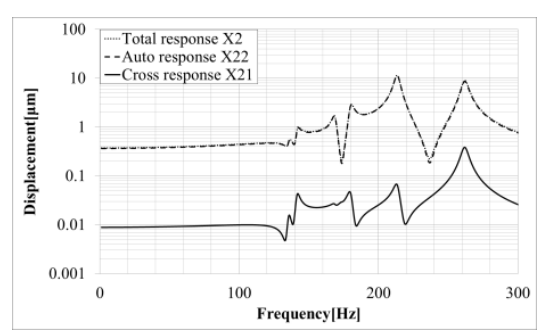

(a) In the $X$-direction

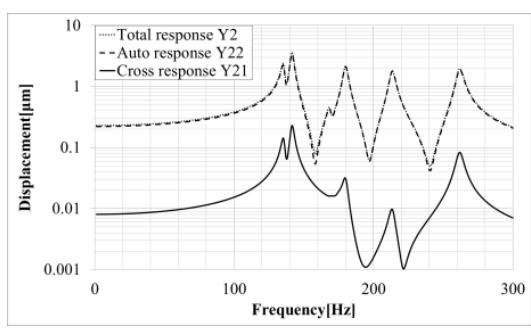

(b) In the $Y$-direction

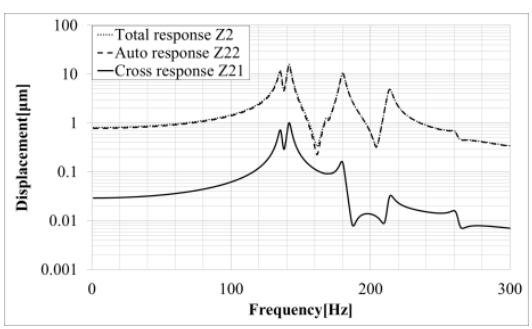

(c) In the Z-direction

Figure 7. Computed harmonic responses, auto- and cross-responses at the $2^{\text {nd }}$ spindle.

Table 2. Percent cross response to total response ratios at the $1^{\text {st }}$ spindle.

\begin{tabular}{|c|c|c|c|}
\hline \multirow{2}{*}{ Percent Static Cross Response to Total Response Ratio, SCR (\%) } & $X$-direction, ${ }^{S C R_{x 12}}$ & $Y$-direction, ${ }^{S C R_{r 12}}$ & Z-direction, ${ }^{S C R_{212}}$ \\
\hline & 2.50 & 0.55 & 0.81 \\
\hline \multirow{2}{*}{ Percent Dynamic Cross Response to Total Response Ratio, DCR (\%) } & $X$-direction, ${ }^{D C R_{x / 2}}$ & $Y$-direction, $D C R_{112}$ & Z-direction, $D C R_{212}$ \\
\hline & 10.59 & 1.17 & 4.19 \\
\hline
\end{tabular}

Table 3. Percent cross response to total response ratios at the $2^{\text {nd }}$ spindle.

\begin{tabular}{|c|c|c|c|}
\hline \multirow{2}{*}{ Percent Static Cross Response to Total Response Ratio, SCR (\%) } & $X$-direction, ${ }^{S C R_{x 21}}$ & $Y$-direction, ${ }^{S C R_{r 21}}$ & Z-direction, ${ }^{S C R_{z 21}}$ \\
\hline & 2.37 & 3.52 & 3.60 \\
\hline \multirow{2}{*}{ Percent Dynamic Cross Response to Total Response Ratio, DCR (\%) } & $X$-direction, $D^{D C R_{x 1}}$ & $Y$-direction, ${ }^{D C R_{r 21}}$ & Z-direction, ${ }^{D C R_{221}}$ \\
\hline & 4.25 & 6.27 & 6.30 \\
\hline
\end{tabular}

\section{Results and Discussion}

From the harmonic response analysis results as shown graphically in Figures $\mathbf{6}$ and $\mathbf{7}$ and the percent SCRs and DCRs summarized in Tables 2 and 3, the percent SCRs at both the $1^{\text {st }}$ and $2^{\text {nd }}$ spindle noses were less than $4 \%$. However the percent DCRs were around $4 \%-7 \%$ at both spindle noses. Furthermore, the biggest percent DCR exceeds $10 \%$ even though the turning lathe had been optimum designed previously. Thus, careful consideration should be given to the effect of cross response on whole (or total) vibration response at each spindle in order to develop or design multi-spindle type multi-functional machine tools.

\section{Concluding Remarks}

In order to analyze how the two spindles affect each other's vibrations in a dual spindle type multi-functional turning lathe when they are doing machining operations simultaneously, in this study, a generalized machine structure model under harmonic forces acting on two different nodes has been introduced and the correlation equations of the structural responses at the two nodes have been derived. Furthermore, the derived correlation equations of the structural responses of the generalized machine structure model have been applied to the dual spindle type multi-functional turning lathe. As the results with FEM structural analysis of the turning lathe, the percent SCR and the percent DCR of each spindle of the multi-functional turning lathe have been obtained; Computed percent SCRs at both spindle noses are less than $4 \%$, but most of computed percent DCRs are around $4 \%-6.3 \%$ and the biggest one reaches about $10.6 \%$. In conclusion, careful consideration should be given to the effect of cross response on whole vibration response at each spindle to develop high precision multi-spindle type machine tools.

\section{Acknowledgements}

This work was supported by the project "Development of a two-spindle and two-turret multi-tasking lathe for a production line supporting the high precision processing for automobile small-parts" (Grant S2071895) sponsored by the small and medium business administration Korea, and AMECO Co., Ltd.

\section{REFERENCES}

[1] T. Moriwaki, "Multi-Functional Machine Tool," CIRP Annals-Manufacturing Technology, Vol. 57, 2008, pp. 736-749.

[2] A. Nagae, "Development Trend of Multi-Tasking Machines," Proceedings of the $11^{\text {th }}$ International Conference on Machine Tool Engineers, 2004, pp. 312-323.

[3] M. Nakaminami, T. Tokuma, M. Moriwaki and K. Nakamoto, "Optimal Structure Design Methodology for Compound Multiaxis Machine Tools, I-Analysis of Requirements and Specifications," International Journal of Automation Technology, Vol. 1, No. 2, 2007, pp. 78-86.

[4] M. Nakaminami, T. Tokuma, K. Matsumoto, S. Sakashita, 
T. Moriwaki and K. Nakamoto, "Optimal Structure Design Methodology for Compound Multiaxis Machine Tools; II-Investigation of Basic Structure,” International Journal of Automation Technology, Vol. 1, No. 2, 2007, pp. 87-93.

[5] Y. H. Choi, S. H. Jang, H. M. Park, S. J. Jang and J. Y. Cho, "A Genetic Algorithm Based Multi-Step Design Optimization of a Machine Structure for Minimum Weight and Compliance,” Proceeding of SICE, 2005, pp. 476-481.

[6] U. Heisel and M. Gringel, "Machine Tool Design Requirements for High-Speed Machining," Annals of the CIRP, Vol. 45, No. 1, 1996, pp. 389-392. http://dx.doi.org/10.1016/S0007-8506(07)63087-X

[7] U. Heisel and A. Feinauer, "Dynamic Influence on Workpiece Quality in High Speed Milling," Annals of the CIRP, Vol. 48, No. 1, 1999, pp. 321-324. http://dx.doi.org/10.1016/S0007-8506(07)63193-X

[8] S. H. Jang, J. H. Oh, H. S. An and Y. H. Choi, "Multi-
Objective Structural Optimization of a Pin Turning Device by Using Hybrid Optimization Algorithm,” Proceedings of the International Conference of Manufacturing Technology Engineers, Seoul, Korea, 2012. p. 73.

[9] S. H. Jang, W. Y. Jeong, B. C. Kwon and Y. H. Choi, “A Study on the Static Optimization of Multi-Axis Milling Machine Using TMSA,” Proceedings of KSPE 2009 Annual Spring Conference, 2009, pp. 227-228.

[10] S. H. Jang, Y. H. Choi and J. S. Ha, “A Study on Analysis of Dynamic Compliance of a 5-Axis Multi-tasking Machine Tool by Using F.E.M and Exciter Test," Transaction of the Korean Society of Machine Tool Engineers, Vol. 18, No. 2, 2009, pp. 162-169.

[11] Y. H. Choi, J. H. Oh, D. H. Kim, T. Y. Seo and K. H. Kim, "Structural Design Optimization of a 2-Spindle 2Turret Type Multi-tasking Lathe," Proceedings of KSME 2013 Annual Spring Conference, Jeju, Korea, 2013, pp. 535-536. 